\title{
Literature Review on the Significance of Death Conferences for Nurses in Japan
}

\author{
Yuko Harding*, Michiyo Ando, Masako Yano \\ Faculty of Nursing, St. Mary's College, Tsubukuhonmachi, Kurume, Fukuoka, Japan \\ Email: *harding@stmaryacjp.onmicrosoft.com
}

How to cite this paper: Harding, Y., Ando, M. and Yano, M. (2019) Literature Review on the Significance of Death Conferences for Nurses in Japan. Open Journal of Nursing, 9, 521-533.

https://doi.org/10.4236/ojn.2019.95044

Received: April 7, 2019

Accepted: May 24, 2019

Published: May 27, 2019

Copyright ( $\odot 2019$ by author(s) and Scientific Research Publishing Inc. This work is licensed under the Creative Commons Attribution-NonCommercial International License (CC BY-NC 4.0). http://creativecommons.org/licenses/by-nc/4.0/

\begin{abstract}
Aim: To identify evidence in the literature on the significance of death conferences for nurses. Method: This is an integrative literature review, consisting of scientific articles published on "Ichushi-Web", an Internet-based retrieval service by the Japan Medical Abstracts Society (JAMAS), PubMed, Medline, Cochrane library, and Google Scholar for 'the whole year,' combining the keywords "death conference", "nurse", and "KJ method". The significance of death conferences was reviewed by comparison of prior qualitative studies analyzed by the KJ method. Result: Seven studies, all from Japanese, were identified. The significance of death conferences showed "refraction", "cooperation", "directionality of nursing", "caring for patient", "caring for family", "specific methods of nursing", and "feelings of nurses". Conclusion: The significance of death conferences is considered to be the effect on nurses for amelioration in the quality of patient and family care, development of human relations, and growth as a nurse. The quality of death conferences should be enhanced based on the guidelines for its clear definition.
\end{abstract}

\section{Keywords}

Death Conference (DC), Nurse, KJ Method

\section{Introduction}

Death conferences, which occur after the death of patients, are when the medical staff (nurses, doctors, and paramedics) gather and reflect on the care for them with a view to improving quality of care. Through discussions, it also encourages the growth of individual nurses [1]. In a ward with many chances to face death, such as in terminal care and palliative care, and sites for emergency medical treatment, death conferences serve as a common setting for nursing care review and learning and sharing the direction of nursing care. However, there is no 
well-known clear definition of death conferences. Moreover, comparative studies of literature relating to the significance of death conferences for nurses have not been seen in Japan. There is no evidence of the comparison of literature about death conferences in qualitative studies using the KJ method [2] [3] [4]. It is possible to improve the quality of patient and family care and encourage the growth of nurses while clarifying the significance of death conferences for nurses, making them more meaningful.

\section{Definitions of the Terms}

For the purposes of this review, the definitions of terms are shown below. Death conference was defined as a place for looking back on the care given to a dying patient or family member. It is intended to lead to better care [5]. It is also aimed at improving the quality of care in the future and supporting the growth of individual nurses [1]. According to Naito, DC is a conference held after a patient's death and discharge, where nurses look back on their patient's progress and memories with their family, grief care, and nursing [6]. Hasegawa and Kobayashi have a different definition of DC where they state that it is a conference to review care performed by the medical team after the death of a patient and to deepen thoughts on terminal care [7] [8]. The KJ method (Kawakita's initials) means a creative information processing method and a solution to a problem invented by Dr. Jiro Kawakita [9]. The KJ method captures the diversity of voices in the field as their individuality. It is a method of structurally assembling the voices and grasping the problem as they speak [3]. This method should be emphasized as a generalized brainstorming technique [2].

\section{Aim}

To conduct a literature review relating to the significance of death conferences for nurses in qualitative studies using the KJ method.

\section{Method}

The review includes the result of observational (cross-sectional/longitudinal) studies which explored the significance of death conferences being a main outcome variable on nurses by comparison of prior qualitative studies analyzed by the KJ method. Through September 2016, the following databases, 'Ichushi-Web,' the Internet-based retrieval service by Japan Medical Abstracts Society (JAMAS) [10], PubMed, Medline, Cochrane library, and Google Scholar were searched by the first author to find additional studies related to the topic, as were the reference lists of included papers and related reviews. Search terms included a combination of keywords by thesaurus, i.e., 'death conference,' 'nurse,' and 'KJ method' in qualitative studies using the KJ method, and the retrieval year was 'the whole year,' and the original articles published were peer-reviewed.

Articles were excluded if they were case reports, the research subjects who were not nurses, not focusing on the significance on the nurses, not with the KJ 
method, interviews, and re-listing of duplicate documents. No further restrictions were made.

Based on the preferred responding items for systematic reviews (PRISMA 2009) [11], which also aim to improve the quality of systematic reviews, the search was performed in the next step. At first, records were identified through searching the database identified above. The first author reviewed the titles of all retrieved articles; titles not meeting the predefined inclusion criteria were excluded. When the authors were uncertain, or if the article was considered relevant, authors reviewed the abstracts and excluded records that did not meet the inclusion criteria. After abstract review, when the authors were uncertain regarding the relevance of an article or if the article was considered relevant, full texts were reviewed independently. In instances of disagreement, authors discussed until a consensus was reached. Next, a search of Google Scholar and scanned titles of reviews identified in the first step, as well as the reference lists of selected papers and current relevant literature, was perfumed (Figure 1).

In terms of analytical method, as for the significance of DC, items to be aimed at each survey and the categories extracted by the KJ method are described. The categories in this research, the categories in each article are reclassified, indicated by [ ] as new main categories, and subcategories are shown in italics.

\section{Results}

\subsection{Trends and Summary of Research}

The selection process of the object to be analyzed and containing search terms and pre-research fraction is shown in Figure 1. Searching for 'death conference' included 92 articles (publication: June 2004-January 2016). Seven domestic papers considered the significance of DC under the KJ method with no overlapping documents. Finally, the seven studies were included in this qualitative research using the $\mathrm{KJ}$ method. As death conferences, it is not a hit in the search for literature in English (using PubMed, Medline, and the Cochrane library).

\subsection{Significance of DC on Nurses}

Even if documents were searched under 'all' conditions, they were only documents between 2007 and 2014 (Table 1). The significance of DCs was divided

Table 1. Significance of DCs analyzed using the KJ method.

\begin{tabular}{l} 
Authors, yr.* \\
\hline $\begin{array}{c}\text { Extraction category } \\
2014[16]\end{array}$ \\
$\begin{array}{ll}\text { 1) Thoughts of nurses who support the decision-making of terminal cancer } \\
\text { 2) Involvement with the patient's family } \\
\text { adachi et al. } & \text { b) Wespect family's wishes } \\
2012[15] & \text { c) Responding to the first meet in a patient's family at End-of-life care }\end{array}$ \\
\hline
\end{tabular}




\section{Continued}

1) Learning how to help terminally ill patients

a) Learning through DCs

b) Nurse's thought towards End-of-life care

c) Nurse's anxiety and confusion

2) Self-reflection

a) Reflection and future relations

b) Skill level raise up

3) Concern with palliative care

a) Learning of palliative care

b) Patient-centered End-of-life care

Koyama et al.

c) Nursing for End-of-life care

2012 [12] d) Worthwhile

4) Team sharing and collaboration

a) Sharing

b) Cooperation

c) Follow-up between staff

d) Team solidarity

5) Collaboration with other professions Staff meeting and exchanges of views

6) Future direction of nursing care Continuing DC

1) An inability to relieve a patient's pain

2) Involvement with patients who have a gap in perceptions of the condition

Ishikawa et al. 3) The act of responding to a patient's emotional expression and questions about 2010 [14] patient's remaining life

4) Dealing with family members of patients who seem to have difficulty involved

5) Involving ethical issues

1) Care review

2) Deepening of human understanding

Kobayashi et al. 3) Fulfillment of a patient's wishes

2010 [8] 4) Challenges for the future

5) Positive opinion for holding DCs

6) Ephemeral feelings for dying patients

1) Significance of expectations for DCs

a) Sharing of values and specific measures

b) Improvement of self-efficacy as a nurse and catharsis

c) Sharing peace of mind, healing through conversation

2) A malfunctioning problem

a) How to provide patient care

Tsuchie et al

b) How to plan (progress) DCs

2010 [11]

3) Specific measures

a) Planning of DCs

b) A way of proceeding with a fixed purpose

c) Considering member structure according to a purpose

d) Respect, trust, and understand companionship

4) Aim

a) Improvement of the practice of nursing in a hospital ward

b) Growth of individual nurses

1) Care review

2) Sharing information and case studies with medical teams

Hasegawa et al.

3) DC learning

2007 [7]

4) View of life and death

5) Expressing emotions

*publishing year; DC: Death Conference. 


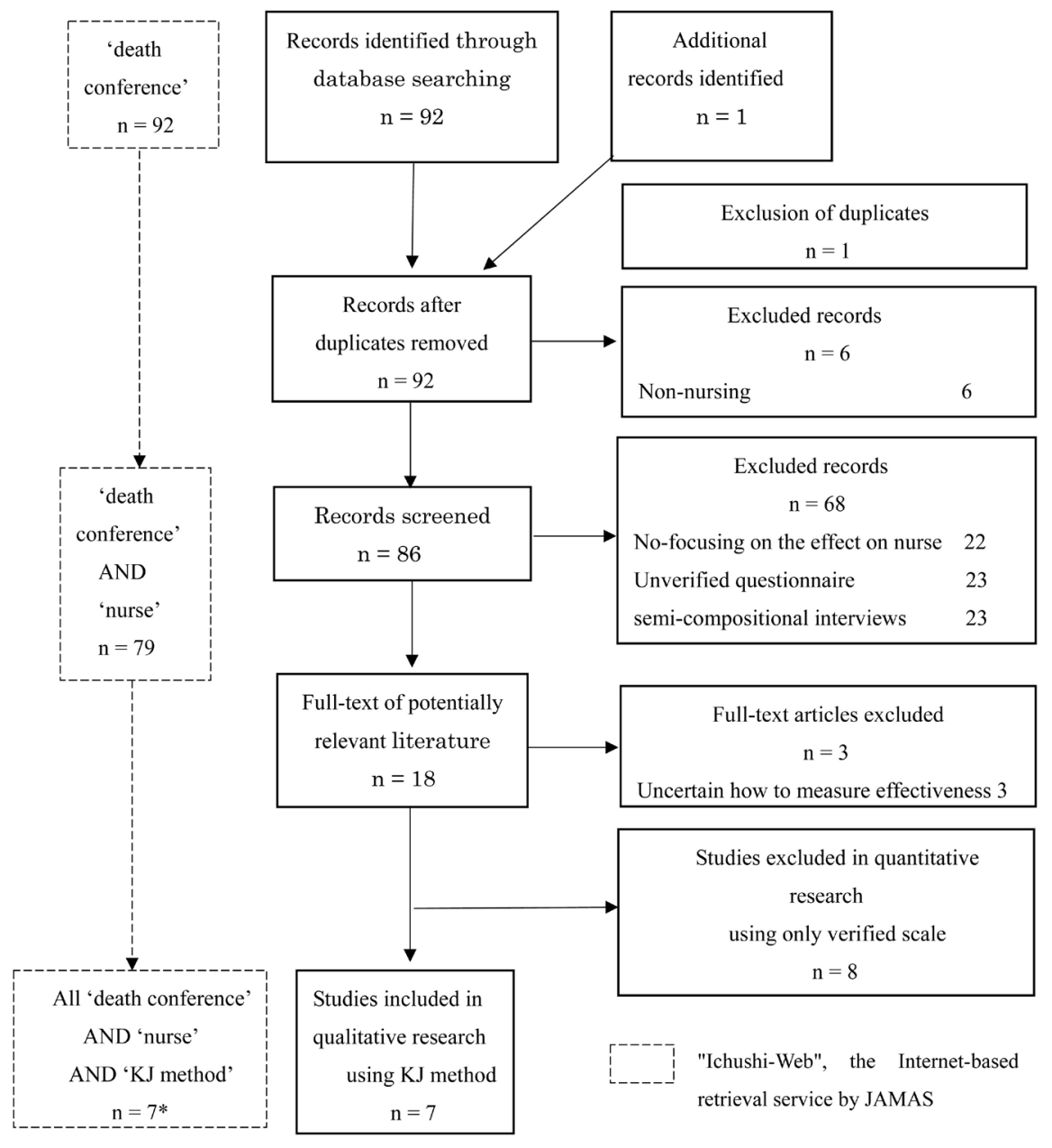

Figure 1. Flowchart of literature search and study selection. ${ }^{\star}$ Note: Search September 27, 2016; there is no thesaurus and no controlling language in terms of vocabulary of 'Death Conference,' each term was multiplied by all, the original, and the search year which was to the full year.

into seven categories. [Reflection] Care review [7] [8] and Self-reflection [12]. [Cooperation] Team sharing and collaboration [12], Sharing information and case studies with medical teams [7], Collaboration with other professions [12], and Deepening of human understanding [8]. The future [Direction] of nursing includes Future direction of nursing care [12], Challenges for the future [8], and Significance of expectations for DC (Sharing of values and specific measures) [13]. [Response to patients] includes An inability to relieve a patient's pain, Involvement with patients who have a gap in perceptions of the condition, and The act of responding to a patient's emotional expression and questions about patient's remaining life [14]. [Response to patients' family] includes Involvement with the patient's family [15], Dealing with family members of patients who seem to have difficulty involved and Involving ethical issues [14]. [Specific measures for nursing] include Learning how to help terminally ill patients [12], DC learning [7], Anxiety of postmortem treatment [15], and Nursing intervention to support decision-making [16]. [Feeling of nurses] includes Thoughts of 
nurses who support the decision-making of terminal cancer patients [16], Fulfillment of a patient's wishes, Positive opinion for holding DC, Ephemeral feelings for dying patients [8], View of life and death, Expressing emotions [7], Improvement of self-efficacy as a nurse and catharsis [13], and Concern with palliative care [12].

\section{Conclusion}

\subsection{Definition of Death Conferences}

As mentioned from the definition of death conferences, the definition included the terms 'nursing/care' and 'look back/review.' The timing of a death conference review is divided into the concept of patient death, when the patient is dying, and post-patient death. No definitions included words about what nurses should do after death conferences.

In the literature search, some of the documents did not include the definition of death conferences. In those cases, it was unclear how each of the writers understood death conferences. In the future, the definitions of death conferences will need to be presented in order to make objective comparisons based on whether or not those states are true. Furthermore, 'death conference' was not a hit in the search for literature in English (using PubMed, Medline, and the Cochrane library), and it is likely that a guideline for definitions on death conferences will be necessary in the future.

\subsection{Significance of DCs}

In the context of DC's significance, DC is seen as a place to share the future [direction] of nursing in the [Reflection] and [Cooperation] of patient care. It is also necessary to consider how to [respond to patients and families] and [specific measures for nursing] for nursing through discussion, and above all, to control [emotion of nurses] (Figure 2). Improving the quality of DCs promotes the improvement of patient care quality and growth as a nurse, leading to the growth of human relationships.

1) [Reflection] Care review-Self-reflection

Hasegawa [7] reported about Care review from an interview with 11 nurses in the mammary surgical ward at a DC where, by looking back on nursing care at DCs, nurses were able to learn about patient behavior that was difficult to understand and how to deal with difficult times. A study of Hasebe [5], a qualitative research that was not found in the search for "KJ method" in "Ichushi-Web" shown in the categorization of the significance of DCs, about [reviewing the practical nursing], which includes subcategories of nursing's difficulty, difficulties in nursing care, reflections of nursing, conflicts in nursing care, and nursing practice, evaluated as good.

In a questionnaire survey on the change in the staff members' behavior by the DC in an acute ward [12], Koyama et al. wrote their reflections, future involvement, and increase in skill in subcategories concerning self-reflection. From the 


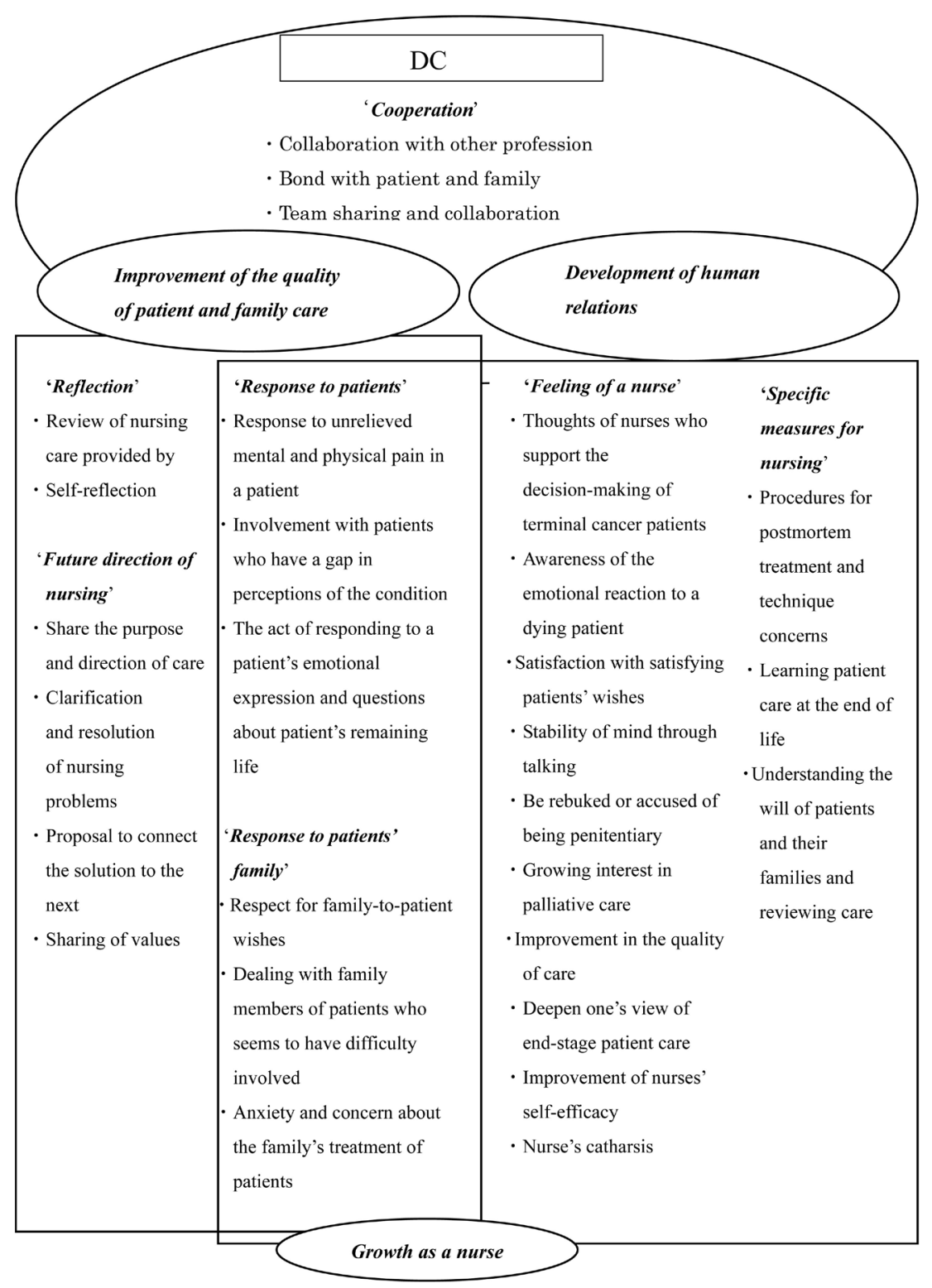

Figure 2. Significance of the conference guided by the KJ method.

above, in DCs, it may be possible to improve the quality of care and increase the motivation to work as a nurse by aiming to improve skills by evaluating and sharing nursing practices that were evaluated to have been good at the same time as the reflection. Care review and self-reflection conducted at DCs seem to improve the quality of patient and family care.

2) [Cooperation] Team sharing and collaboration, Collaboration with other professions, Sharing information and case studies with medical teams, and Deepening of human understanding.

Koyama et al. in his study indicated the effectiveness of sharing and cooperation. Among teams in DCs from the nurses' opinions that talked about their problems, they felt encouragement and compassion among the nurses, and their teams grew more united [12]. Koyama et al. wrote Staff meeting and exchanges 
of views in subcategories concerning Collaboration with other professions.

Hasegawa reported that all 11 nurses at the mammary surgical ward replied before the DC that they felt 'insufficient information sharing and collaboration with doctors,' but at the post-DC interview, everyone was glad to have done it. Moreover, by analyzing the DC word-per-word, the researchers reported a change in the field of nurses' opinions that they could recognize the amount of information each person had and confirm the importance of collaboration. The author describes the significance of the DC as a motivator to build close ties with the medical teams and as an opportunity to learn about each other from many different types of work [7]. Therefore, it seems that DC is an essential place to share the mutual learning of medical personnel, deepen understanding, and build trust among team members. DCs have important implications for Collaboration with other professions, increase patient-family ties, and strengthen sharing and collaboration within a team.

3) The future [Direction] of nursing Future direction of nursing care, Challenges for the future, and Significance of expectation for DC (Sharing of values and specific measures).

Koyama et al. have cited Continuing $D C$ as a subcategory for Future direction of nursing care [12]. Kobayashi et al. suggested in his study that in the comments presented at DCs, they hope the participants desire to participate in study meetings and would like to strengthen their cooperation with doctors as Challenges for the future [8]. Tsuchie et al. tell in the view of the nurses' DC in the chronic medical ward, as Significance of expectation for DC (Sharing of values and specific measures) that they want the participants to not only reflect but also to analyze their problems and obtain solutions to connect [13]. Thus, in order to enhance collaboration with various types of jobs, such as doctors, DCs should continue to analyze nursing problems and clarify the direction of nursing. In DCs, future direction of nursing, such as sharing the purpose and direction of care, clarification and the guidance of solutions of nursing problems, proposal to connect one solution to the next, the sharing of values, and the directionality of nursing, are likely to lead to a reversal of the patient and to improve the quality of patient and family care.

4) [Response to patients] An inability to relieve a patient's pain, Involvement with patients who have a gap in perceptions of the condition, and The act of responding to a patient's emotional expression and questions about patient's remaining life.

Stresses in nursing care of these nurses need to be expressed in concrete terms at DCs and supported by staff to reduce stress on the staff. In case the nurse has stress concerning unrelieved mental and physical pain in a patient, involvement with patients who have a gap in perceptions of their condition, and the act of responding to a patient's emotional expression and questions about the patient's remaining life, DCs have the significance as a place to present their stressful situations and share information. Moreover, understanding the status quo by the 
team can deepen the bonds between patients and family members and improve the quality of patient and family care.

5) [Response to patients' family] Involvement with the patient's family, Dealing with family members of patients who seem to have difficulty involved, and Involving ethical issues.

From a questionnaire survey of nurses for the introduction of DCs, Adachi et al. point out subcategories of Involvement with the patient's family: Respect family's wishes, Worry about the behavior and expressions of the patient's fami$1 y$, and Responding to the first meet in a patient's family at End-of-life care. They report that nurses were worried about when to talk to a patient's family, how to explain, and what to say [15].

Ishikawa et al., report concerning dealing with family members of patients who seems to have difficulty involved, stressful situations in which family members' aggressive feelings, speech, and behavior change, and when family members cannot accept the patient's condition to the end. They also described Involving ethical issues, the stress of having nurse calls that are often made during night shifts when there are fewer nurses and the stressful feeling about whether it was really good to have detained a patient and sedated him because of delirium [14]. Thus, the nurse's confusion over family speech and behavior should not be left to the nurse's competence but should be shared by the team to better understand and respond to the situation. This may reduce the stress of nurses dealing with patient families on the spot, and lead to improve the quality of patient and family care by diffusing the situation.

A study of Hasebe [5] has shown in the categorization of the significance of DCs, in [Response to patients' family], raising the thought and evaluation of patients and family which includes subcategories of negative/positive feelings toward patients and families, analysis of patient's thoughts, and analysis of family feelings. The results of the qualitative studies above show the significance of DCs as a place in a team where nurses care for patients and families express their feelings for them. It will also be necessary to analyze the quality of the content of DCs in order to accept nurses' thoughts and improve patient family care. Discussions at DCs on respect for family-to-patient wishes, dealing with family members of patients who seems to have difficulty involved, and response to patients' family such as anxiety and concern about the family's treatment of patients, can improve the quality of patient and family care.

6) [Specific measures for nursing] Learning how to help terminally ill patients, DC learning, Anxiety of postmortem treatment, and Nursing intervention to support decision-making.

In a survey of 19 nurses at a DC on how they feel to support decision-making before dying for terminal cancer patients and their families, the decision-making support provided by nurses suggests "Continue to keep track of the patient's family's mood since the decision was made in daily communication and support through team medical care" [16]. Koyama et al. cites the subcategories of Learn- 
ing through DC, Nurse's thought towards End-of-life care, and Nurse's anxiety and confusion in learning how to help terminally ill patients [12]. Adachi et al. report regarding Anxiety of postmortem treatment, nurses' anxiety about postmortem procedures and technology [15]. As mentioned above, DCs are a valuable place to confirm how to help patients improve patient care. Tsuchie et al. mentioned in a survey of 20 nurses in the terminal care ward who participated in the conference the subcategories of Planning of $D C$, $A$ way of proceeding with a fixed purpose, considering member structure according to a purpose, and Respect, trust and understand companionship in Specific measures [13]. It lists proposals for creating a comfortable-to-talk atmosphere, an environment that provides confidence, respect, trust, and understanding for the DC project. For DCs to work, they propose consideration of how to provide patient case and how to plan (progress) DCs. Hasegawa et al. notes about DC learning, before DCs that 'Nurses are not confident about terminal care and lack of knowledge,' however, after DCs, they were able to gain knowledge from other experts, such as 'learning about pain relief from pharmacists' and hind meaning in care they didn't have [7]. In DCs, consideration of specific measures for nursing, including confirming procedures for postmortem treatment and technical concerns, learning patient care at the end of life, understanding the will of patients and their families and reviewing care promote growth as a nurse.

7) [Feeling of nurses] Thoughts of nurses who support the decision-making of terminal cancer patients, Fulfillment of a patient's wishes, Positive opinion for holding DCs, Ephemeral feelings for dying patients, View of life and death, EXpressing emotions, Improvement of self-efficacy as a nurse and catharsis, and Concern with palliative care.

In post-DCs, Koyama et al. cited sub-categories of Learning of palliative care, Patient-centered End-of-life care, Nursing for End-of-life care and Worthwhile about concern with palliative care. They also said from their study that Nurses have come to think about what they should do from the heart of their patients and their families, felt a sense of challenge and achievement, have been able to face their life and are proud of their job [12]. Kobayashi et al. note that DCs have raised nurses' awareness of care, as shown below. They say that about Fulfillment of a patient's wishes, nurses realized the effect from the results of creating an environment where a patient could spend time with family and assisting patients to return home temporarily. They also discuss about Positive opinion for holding DCs, nurses hoped to participate and have more discussions with each other in DCs. They also note about the Ephemeral feelings for dying patients, expression of emotion or thought of nurses to dying patients or those who want more medical treatment [8]. Tsuchie et al., as the significance of DCs cited Improvement of self-efficacy as a nurse and catharsis, sharing peace of mind, healing through conversation as subcategories of Significance of expectations for DC and tells nurse's opinion that someone who feels the same feeling can be reassured [13]. Thus, nurses' ephemeral feeling for the dying patients should be expressed at DCs with medical team members composed of other professional 
members, and supported by DCs, so that individuals are able to improve their sense of self-effects, get catharsis and take care of them positively without losing sight themselves. If nurses' feelings are controlled well, it will help to grow as a nurse.

Thoughts of nurses who support the decision-making of terminal cancer patients, awareness of the emotional reaction to a dying patient, satisfaction with satisfying patients' wishes should be shared at DCs in order to stabilize the mind through talking. DCs should not be a place where nurses are denied and blamed through their constant reflection. DCs can raise nurses' interest in palliative care, improvement in the quality of care and deepen their view of end-stage patient care. Reviewing the situation regarding improvement of nurse's self-efficacy and their catharsis by the team's support will lead to their growth of human relationships.

\subsection{Future Challenges}

Nurses are expected to improve patient and family care, encourage their growth as nurses and human relationships, and maintain their motivation for nursing. For this reason, it will be necessary to reflect on terminal care and nursing at DCs. In the future, quantitative research on the effect of DCs on nurses, with self-evaluation of nursing at Terminal Care and DCs especially. Moreover, it is necessary to make guidelines on the definition of DCs and to develop an index or an evaluation scale capable of objectively measuring the effect of DCs on a nurse, concretely, nurses' growth and change of behavior, mood, and the quality of care for patients.

\section{Summary}

1) This is an integrative literature review clarifying the definition and the significance of death conferences reviewed by comparison of seven prior studies analyzed mainly by the KJ method in the Internet-based retrieval service by Japan Medical Abstracts Society (JAMAS);

2) DCs are seen as a place to share the future [direction] of nursing in the [Reflection] and [Cooperation] of patient care. It is also necessary to consider how to [respond to patients and families] and [specific measures for nursing] for nursing through discussion, and above all, to control [emotions of nurses];

3) Improving the quality of DCs promotes the improvement of patient care quality and growth as a nurse, leading to the growth of human relationships.

\section{Conflicts of Interest}

The authors declare no conflicts of interest regarding the publication of this paper.

\section{References}

[1] Hiroko, H. (2011) Grief Care for Family and Nurses. Igaku-Shoin Ltd., Tokyo. https://iss.ndl.go.jp/books/R100000002-I000011224361-00 
[2] Kawakita, J. (1967) Hasso Hou (Idea Generation Method). Chukoshinsho, Chuokoron-Shinsha, Inc., Tokyo. https://iss.ndl.go.jp/books/R100000039-I001224315-00

[3] Kawakita, J. (1970) Zoku Hasso Hou (Idea Generation Method, Continued). Chukoshinsho, Chuokoron-Shinsha, Inc., Tokyo. https://iss.ndl.go.jp/books/R100000002-I000001185938-00

[4] Kawakita, J. (1986) The KJ Method: Seeking Order Out of Chaos. Chuokoron-Sha, Tokyo. https://iss.ndl.go.jp/books/R100000002-I000001831062-00

[5] Hasebe, M., Taguchi, M. and Tsuge, M. (2012) Effect of Death Conferences as Seen from Changes in Nurses' Comments. Nihon Kango Gakkai Rombunshuu, Kango Sougou, 42, 218-221. https://iss.ndl.go.jp/books/R000000004-I023617204-00

[6] Naito, C., Nakai, O., Omote, S., Yabuta, Y., Hoshino, T. and Miyahata, T. (2009) The Effect of Death Conferences in terminal Care; Evaluation of Stress Reduction Using POMS. Nihon Kango Gakkai Rombunshuu, Kango Kanri. 39, 330-332. https://jglobal.jst.go.jp/en/detail?JGLOBAL ID=200902260488192837

[7] Hasegawa, M., Eto, A. and Yamauchi, N. (2007) Impact of Death Conference in the Medical Team on Nurses' Self-Efficacy. Nihon Kango Gakkai Rombunshuu, Kango Sougou, 38, 49-51. https://jglobal.jst.go.jp/en/detail?JGLOBAL ID=200902285011484057

[8] Kobayashi, M. and Shibuya, M. (2010) Consideration of Terminal Care in Our Hospital Ward. By introducing Death Conferences. Pre Symptomatic Medicine and Anti Aging, 19, 143-145. https://jglobal.jst.go.jp/en/detail?JGLOBAL ID=201002214228207333

[9] Kabaya, H. (1989) Improving Student's Japanese Spoken and Written Expression Using an Application of the KJ-Method. Bulletin of Center for Japanese Language, Waseda University, 1, 65-84.

https://waseda.repo.nii.ac.jp/?action=pages view main\&active action=repository view main item detail\&item id=26046

[10] JAMAS (2019). https://www.jamas.or.jp/english/

[11] PRISMA (2015). http://www.prisma-statement.org/

[12] Koyama, K., Kazama, K., Nakamura, M., Aonuma, E. and Yoshikawa, K. (2012) Behavioral Change of Staff by Death Conferences in Acute Care Ward-Aiming at Improvement of Quality of Terminal Care. Naganoken Kango Kenkyuu Gakkai Rombunshuu, 32, 79-81.

https://jglobal.jst.go.jp/en/detail?JGLOBAL ID=201202259594399200

[13] Tsuchie, K., Sasaki, M., Kumagai, N. and Ishibashi, T. (2010) Analysis of Way of Grasping Death Conferences by Nurses in Chronic Medical Ward and Consideration of Effective Progress. Nihon Kango Gakkai Rombunshuu, Kango Sougou, 40, 425-427. https://jglobal.jst.go.jp/en/detail?JGLOBAL ID=201002206420128002

[14] Ishikawa C. and Hashizume T. (2010) Nurse's Stress in Palliative Care Seen from Death Conferences. The Medical Journal of Akita City Hospital, 19, 1-5. https://jglobal.jst.go.jp/en/detail?JGLOBAL ID=201002226948889952

[15] Adachi, K. and Tashiro, M. (2012) Deployment of Death Conferences and Nurse's Mind. From a Survey of Nurses for the Introduction. The Japanese Psychiatric Nursing Association, 55, 550-551. https://jglobal.jst.go.jp/en/detail?JGLOBAL ID=201202226647441608

[16] Tanaka, H., Moriya, A. and Sakiko, K. (2014) A Thought of a Nurse Supporting an Endterm Cancer Patient's Decision-Making: Nursing Which Nestles up to the Pa- 
tient Family Who Passes the Time of the Last Moment Is Considered. Annals of Nursing Research, Shinshu University Hospital, 42, 38-40.

https://ci.nii.ac.jp/naid/40020282208

\section{Statement of Authorship}

Category 1

a) Conception and Design: Harding Yuko;

b) Acquisition of Data: Harding Yuko, Michiyo Ando, Masako Yano;

c) Analysis and Interpretation of Data: Harding Yuko, Michiyo Ando, Masako Yano.

\section{Category 2}

a) Drafting the Article: Harding Yuko;

b) Revising for Intellectual Content: Harding Yuko, Michiyo Ando, Masako Yano.

\section{Category 3}

Final Approval of the Completed Article: Harding Yuko, Michiyo Ando, Masako Yano. 